\title{
Blur-modulated orientation perception in the rod-and-frame task
}

\author{
SHELDON M. EBENHOLTZ \\ University of Wisconsin, Madison, Wisconsin
}

\begin{abstract}
In order to assess the role of blur in a rod-and-frame task, an afocal blurring technique was developed that restricted the blur to the frame. Three levels of blur were investigated, along with a nonblurred, directly viewed frame. Results showed a significant drop in the rod-and-frame effect (RFE) with increasing blur (decreasing spatial frequency), but spatial frequencies even as low as $.092 \mathrm{cpd}$ failed to reduce the RFE to zero. Decreasing luminance was correlated with increased blur, but control studies showed that variation in luminance levels between .007 and $.0015 \mathrm{~cd} \mathrm{~m}^{-2}$ had no effect on RFE. The finding of a spatial frequency dependency in the rod-andframe task permits the development of a neuropsychological theory of individual and gender differences typically found in studies of static spatial orientation.
\end{abstract}

In the rod-and-frame task, an observer typically views a large square luminous frame rotated in the frontal plane to some angle relative to the gravitational direction. Adjustments by the observer of a luminous rod, about an axis at the frame center, reliably yield an error in the position of an apparently vertical line in the direction of the tilted frame. The measure of this error represents the rodand-frame effect (RFE) first investigated by Witkin and Asch (1948).

Although performance on the rod-and-frame task is thought to be related to individual differences in cognitive style (Witkin \& Goodenough, 1981), the RFE itself is remarkably insulated from cognitive influence. Thus, it is the retinal, and not the perceptual, size of the frame that influences the RFE (Ebenholtz, 1977; Ebenholtz \& Callan, 1980); a square form organization or a frame-like quality of the surround fails to serve as a surrogate for a complete frame (Streibel, Barnes, Julness, \& Ebenholtz, 1980); and depth separation between line and frame is ineffective in reducing the RFE (Ebenholtz \& Glaser, 1982). These results indicate that stages of processing in which form, size, and depth are extracted, are not implicated in the RFE. Given the further evidence of an influence of the tilted frame on apparent body orientation (Ebenholtz \& Benzschawel, 1977; Sigman, Goodenough, \& Flannagan, 1979), and the fact that the RFE increases with increasing retinal size of the frame, thereby implicating the retinal periphery, a new hypothesis about the RFE seems warranted: The phenomenon represents an instance of a class of visual-vestibular interactions of the type productive of vection, that is, induced self-motion and ap-

This research was supported by Grant BNS 8201411 from the National Science Foundation. Thanks are extended to Jim Dannemiller for his thoughtful comments and to John W. Utrie, Jr., for his aid in directing the experiments.

The author's mailing address is: Department of Psychology, University of Wisconsin, 1202 West Johnson Street, Madison, WI 53706. parent body tilt (Dichgans \& Brandt, 1978). Since peripheral retinal stimulation plays a critical role both in roll vection (e.g., Reason, Mayes, \& Dewhurst, 1982) and in the RFE, it seems plausible to propose that the RFE be regarded as the static analog of vection, contributing to the control of body posture and egocentric orientation via the angular orientation of images represented in peripheral visual stimulation.

Recent research on circular vection (i.e., induced selfmotion around the vertical axis) has provided evidence that indices of vection, such as latency to onset of induced self-motion and the duration of the movement sensation on removal of stimulation, are quite independent of both luminance level and blur, the latter equivalent to about 16 diopters (D) of refractive error (Leibowitz, Rodemer, \& Dichgans, 1979). These results point to the highly functional nature of vection, in that even greatly degraded visual stimulation remains adequate to support dynamic spatial orientation and the sense of ego motion.

The question for the present study is whether the RFE also is robust with respect to optical blur. Accordingly, bearing in mind the significance of peripheral retinal stimulation, the role of blur in the RFE was examined through the use of an afocal blur technique that restricted the blur to the frame, leaving the rod-image intact. Experiment 1 compared a normal frame with one seen under three levels of blur. Since blur reduced peak luminance, Experiment 2 compared the RFE under two luminance levels to determine whether differences obtained in the first experiment might actually be attributable to differences in luminance. In Experiment 3, frames either were nonblurred but differed in luminance or differed in blur but were equated in luminance.

\section{Apparatus \\ The same rod and frame, made of electroluminescent panels, was used in all three experiments. The dimensions were $35.6 \times .8 \mathrm{~cm}$}

GENERAL METHOD 
for each side of the frame; the rod was $7.1 \times .2 \mathrm{~cm}$. One side of the frame thus represented $36.8^{\circ}$ of arc at the retina; the line length equaled $7.6^{\circ}$. The frame was always at a distance of $53.5 \mathrm{~cm}$ from the observer, whose head was stabilized and whose eye level was adjusted to the height of the axis of rotation of the rod at the center of the frame.

An afocal blur of the frame was produced by interposing between the observer and the frame a frosted acrylic panel of the kind commonly used to reduce glare in hung pictures. An aperture of $6.8 \mathrm{~cm}$ at the center of the blur panel permitted a direct view only of the line, which was seen in clear focus, whereas the frame was viewed only after light rays had passed through the light-scattering medium. Magnitude of blur was modulated by altering the distance between the frame and panel and by increasing the thickness of the panel (1 $\mathrm{mm}$ in Experiment 1,2 $\mathrm{mm}$ in Experiment 3).

\section{Procedures}

In all experiments, the RFE was measured by having observers set the rod to the egocentrically defined vertical, as indicated by the chin to forehead or 6 to 12 o'clock direction. Settings were made from two rod starting positions at $25^{\circ}$ clockwise (cw) and counterclockwise (ccw) of true vertical. Judgments of the apparent vertical always were taken first in the absence of the surrounding frame and second with the frame present. Frame tilt was always $22.5^{\circ}$ $\mathrm{cw}$, and viewing was monocular with the dominant eye in Experiments 1 and 2 and binocular in Experiment 3. The measure of the RFE was taken as the algebraic difference in mean settings between frame-absent and frame-present conditions.

\section{Subjects and Design}

The subjects were 64 college students who had volunteered for the study. There were 24 in Experiment 1, 16 in Experiment 2, and 24 in Experiment 3 . In each experiment and in each condition, half the subjects were of each gender. Because of the presence of reliable individual differences in the RFE, all experiments used a within-subjects design, with the order of treatments counterbalanced over subjects.

\section{EXPERIMENT 1}

\section{Method}

Procedure. The RFE was measured first with direct view of the frame, no blur (NB) present. Three blur conditions then followed in counterbalanced order. In the low-blur (LB) condition, the acrylic panel was placed at $5.8 \mathrm{~cm}$ in front of the luminous frame. Two panels, one at $5.8 \mathrm{~cm}$ and a second at $22.6 \mathrm{~cm}$ from the frame were used to produce the medium-blur (MB) condition. High blur (HB) was created by a two-panel sandwich, the side nearest the frame being at a distance of $33.5 \mathrm{~cm}$ from it.

Blur measurement. In order to determine the actual luminance distribution produced at the panels, the projection of one vertical edge of the frame was scanned horizontally with a Photoresearch spotmeter and luminance levels were taken at 1-mm intervals. Readings ceased when brightness levels were less than or equal to .0001 Nit two steps in a row. The results are represented in Figure 1. Inspection revealed that the luminance distributions were essentially Gaussian. Logically, this is to be expected from the convolution of a Gaussian blurring function and a step function, the latter corresponding to the change in luminance at one edge of the frame. Thus, it can be concluded that the effect of the panels was to distribute the incident light energy normally. A second effect was the reduction in peak luminance that necessarily occurred with increasing spread of blur. Considering the three blur conditions, frame-to-panel separations were manipulated so that peak luminance and the bandwidth, measured at one-half of peak amplitude, both produced evenly spaced intervals of about .30 units in logarithmic coordinates.

Spatial frequencies. Given that the luminance is modulated according to a Gaussian function, it is possible to specify the spatial frequency of the pattern corresponding to a side of the frame in each of the blur conditions. Since the ordinate of the unit normal curve at the mean equals .399 , the ordinate height at one-half peak amplitude equals .200 , which corresponds to $1.175 \sigma$ from the mean. If the total function is assumed to span $\mathrm{M} \pm 3 \sigma,{ }^{1}$ then the function at half-amplitude covers $1.175 / 3 \times 2$, or $78.4 \%$ of the total band-

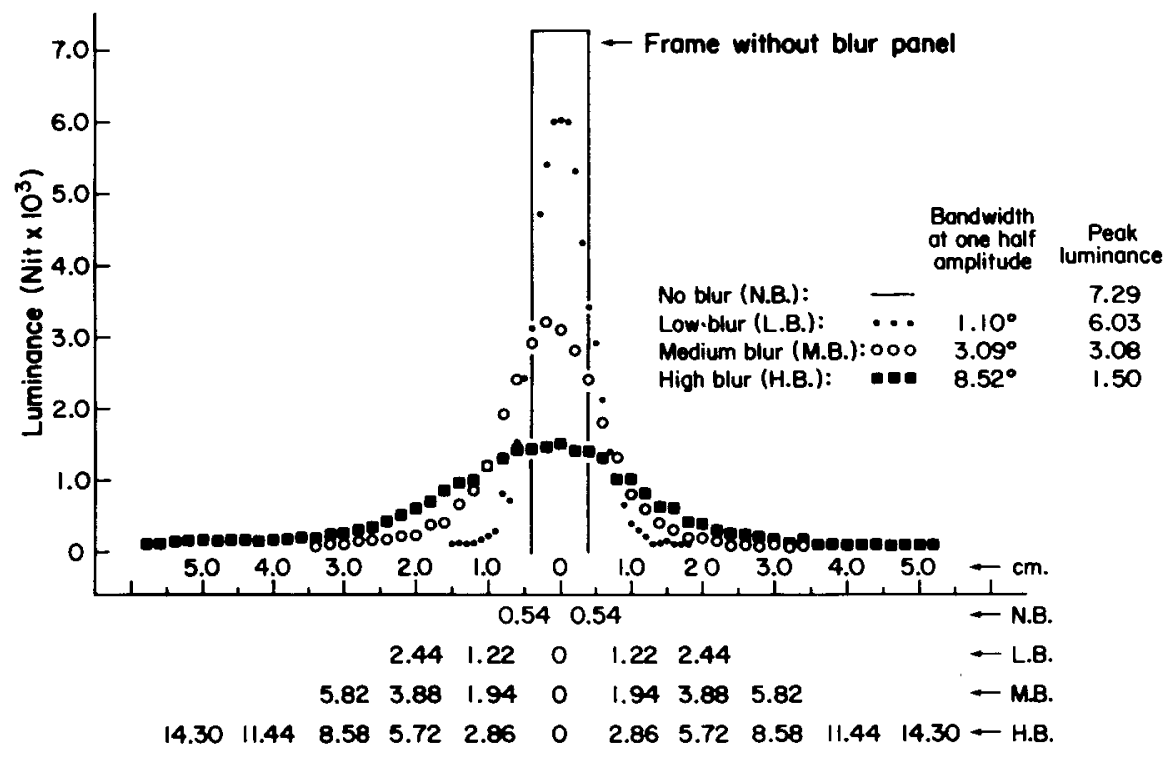

Retinal subtense of luminous target (deg. of arc)

Figure 1. Luminance distributions through a horizontal cross section of one side of the frame. Differing distances between the observer and blur panel caused variation in the retinal projection of the linear extent of each blur pattern. 
Table 1

Rod-and-Frame Effect (Degree) Under Conditions of No Blur (NB), and Low Blur (LB), Medium Blur (MB), and High Blur (HB)

\begin{tabular}{|c|c|c|c|c|c|c|c|c|}
\hline & \multicolumn{2}{|c|}{ NB } & \multicolumn{2}{|c|}{ LB } & \multicolumn{2}{|c|}{ MB } & \multicolumn{2}{|c|}{ HB } \\
\hline & $\mathbf{M}$ & $\sigma_{M}$ & $\mathbf{M}$ & $\sigma_{\mathrm{M}}$ & $\mathrm{M}$ & $\sigma_{M}$ & $\mathrm{M}$ & $\sigma_{\mathbf{M}}$ \\
\hline $\begin{array}{l}\text { Entire Group } \\
\text { Highest Tercile } \\
\text { Lowest Tercile }\end{array}$ & $\begin{array}{l}3.49 \\
6.92 \\
0.06\end{array}$ & $\begin{array}{l}.63 \\
.60 \\
.32\end{array}$ & $\begin{array}{l}3.82 \\
7.09 \\
1.83\end{array}$ & $\begin{array}{r}.73 \\
1.58 \\
.33\end{array}$ & $\begin{array}{l}1.65 \\
3.28 \\
0.27\end{array}$ & $\begin{array}{l}.36 \\
.57 \\
.36\end{array}$ & $\begin{array}{l}1.60 \\
3.08 \\
0.33\end{array}$ & $\begin{array}{l}.42 \\
.86 \\
.37\end{array}$ \\
\hline $\begin{array}{l}\text { Peak Luminance (Nit) } \\
\text { Bandwidth at } 1 / 2 \text { Amplitude }\end{array}$ & \multicolumn{2}{|c|}{.007} & \multicolumn{2}{|c|}{.006} & 3.0 & 03 & & $8.52^{\circ}$ \\
\hline
\end{tabular}

width. Thus, the full bandwidth equals the spread at half amplitude $\times 1.276$. Based on these computations, the frequencies in Gaussian cycles $\mathrm{deg}^{-1}$ were $.715, .253$, and .092 for the low-, medium-, and high-blur conditions, respectively. ${ }^{2}$ In order to achieve a comparable estimate for the NB condition, the lightmeter was used to obtain a luminance profile of the frame edge in the absence of any intervening blur panel. Based on computation of the bandwidth at half-amplitude $\left(.91^{\circ}\right)$, full bandwidth equaled $1.16^{\circ}$, and the corresponding spatial frequency, therefore, was $.862 \mathrm{cpd}$.

\section{Results}

Comparison of the mean RFE over all four conditions showed significant differences $[\mathrm{F}(3,29)=11.98$, $p<.01]$. Separate analyses of the eight subjects who scored highest and the eight who scored lowest on the noblur condition also indicated significant effects $[F(3,21)$ $=3.51$ and 6.83 , respectively, $\mathrm{p}<.01$ in each case].

The pattern of differences in the entire group and in the group of high scorers shows considerable differences between NB and LB, on the one hand, and MB and HB, on the other (see Table 1). Clearly, the RFE diminishes with increasing blur. The pattern for the lowest eight scorers is difficult to evaluate, since only the LB condition produced a mean RFE that was significantly different from zero. Nevertheless, an analysis in terms of spatial frequencies may shed further light on these results.

Figure 2 represents the mean RFE as a function of spatial frequency ${ }^{3}$ for the entire group and also for the eight highest and eight lowest scorers classified according to performance in the NB condition. Linear functions derived from a least squares procedure provided excellent fits for all but the lowest scorers. In this group, there was essentially no RFE at any point except for LB. It cannot be ascertained whether Condition LB is anomalously high or Condition NB anomalously low. In any event, low scorers are relatively less influenced by the frame than are high scorers throughout the range of frequencies tested.

\section{EXPERIMENT 2}

According to the luminance profiles shown in Figure 1, spatial frequency varied directly with peak luminance. Hence, it had to be determined whether the increase in RFE associated with increasing spatial frequency actually was an artifact of increasing peak luminance. Experiment 2 examined this issue by comparing the RFE in two nonblurred or standard frame conditions under two luminance levels. These corresponded to the highest and lowest levels in Experiment 1, namely Condition NB at $.007 \mathrm{~cd} \mathrm{~m}^{-2}$ and Condition $\mathrm{HB}$ at $.0015 \mathrm{~cd} \mathrm{~m}^{-2}$.

\section{Results}

Differences in RFE under high and low luminance approximating the extreme luminance levels of Experiment 1 were without significance $[t(15)=.42, p>.05]$. Furthermore, as Table 2 suggests, neither those highly influenced nor those influenced hardly at all by the tilted frame performed differentially as a function of luminance level. It is clear that differences obtained in Experiment 1 cannot be attributed to correlated differences in peak luminance.

\section{EXPERIMENT 3}

In Experiments 1 and 2, the RFE was obtained under monocular viewing with the dominant eye. Furthermore, the evidence ruling out luminance differences was obtained from subjects who were different from those who, in Experiment 1, had demonstrated a frequency-dependent RFE. Experiment 3 incorporated binocular viewing while the same set of 24 subjects was exposed to a standard non-

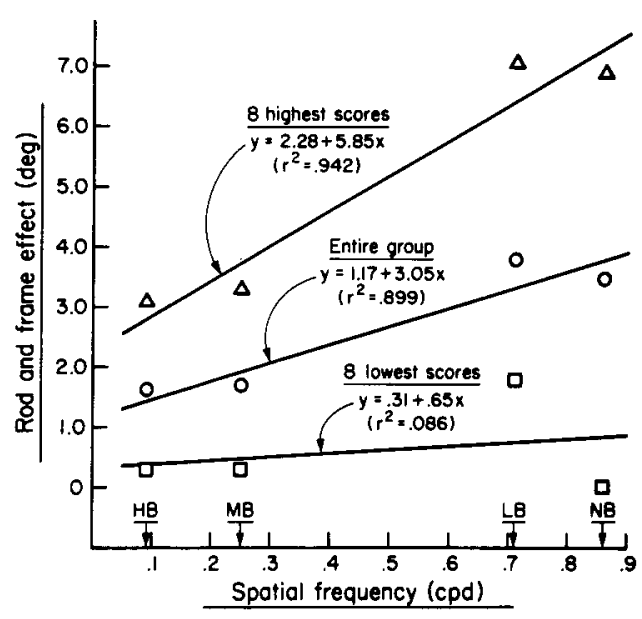

Figure 2. Rod-and-frame effect as a function of spatial frequency of one side of the frame. 
Table 2

Rod-and-Frame Effect Under Normal (.007) and Low (.0015) Luminance (Nit)

\begin{tabular}{lccccc}
\hline & \multicolumn{2}{c}{ Normal } & & \multicolumn{2}{c}{ Low } \\
\cline { 2 - 3 } \cline { 5 - 6 } & $\mathrm{M}$ & $\sigma_{\mathrm{M}}$ & & $\mathrm{M}$ & $\sigma_{\mathbf{M}}$ \\
\hline Entire Group & 2.70 & 0.71 & & 2.86 & 0.85 \\
Upper Half & 4.54 & 1.03 & & 5.18 & 1.16 \\
Lower Half & 0.86 & 0.35 & & 0.53 & 0.48 \\
\hline
\end{tabular}

Table 3

Rod-and-Frame Effect Under Combinations of Standard (S) and Blurred (B) Frame and Standard (.007 Nit) and Reduced (R) Luminance (.003 Nit)

\begin{tabular}{|c|c|c|c|c|c|c|}
\hline & \multicolumn{2}{|c|}{ SS } & \multicolumn{2}{|c|}{ SR } & \multicolumn{2}{|c|}{ BR } \\
\hline & $\mathbf{M}$ & $\sigma_{\mathrm{M}}$ & $\mathbf{M}$ & $\sigma_{\mathbf{M}}$ & $\mathbf{M}$ & $\sigma_{M}$ \\
\hline $\begin{array}{l}\text { Entire Group } \\
\text { Upper Tercile } \\
\text { Lower Tercile }\end{array}$ & $\begin{array}{r}5.61 \\
10.96 \\
1.48\end{array}$ & $\begin{array}{l}0.93 \\
1.29 \\
0.24\end{array}$ & $\begin{array}{r}5.06 \\
10.26 \\
1.29\end{array}$ & $\begin{array}{l}0.90 \\
1.03 \\
0.48\end{array}$ & $\begin{array}{l}2.54 \\
5.48 \\
0.66\end{array}$ & $\begin{array}{l}0.65 \\
1.38 \\
0.37\end{array}$ \\
\hline $\begin{array}{l}\text { Peak Luminance } \\
\text { Bandwidth at } 1 / 2 \text { Amplitude }\end{array}$ & $\begin{array}{l}.0 \\
.9\end{array}$ & .007 & $\begin{array}{l}.00 \\
.91\end{array}$ & & & $\begin{array}{l}03 \\
50^{\circ}\end{array}$ \\
\hline
\end{tabular}

blurred frame at standard luminance of $.007 \mathrm{~cd} \mathrm{~m}^{-2}$ (Condition SS), a standard frame at reduced luminance of $.003 \mathrm{~cd} \mathrm{~m}^{-2}$ (Condition SR), and a blurred frame at reduced luminance (Condition BR). The blur pattern corresponded to a Gaussian spatial frequency of $.218 \mathrm{cpd}$, slightly lower than that of Condition MB in Experiment 1.

\section{Results}

Analysis of variance provided evidence of significant differences among the three means of the entire group $[\mathrm{F}(2,46)=23.24, \mathrm{p}<.01]$ and also among the means of the highest eight scorers $[F(2,14)=23.07, p<.01]$. Low scorers showed a pattern similar to those of others, but effects were not statistically significant $[F(2,14)=$ $1.27, p>.05]$. As the data of Table 3 suggest, only those differences among means that involved the blurred frame (i.e., Condition BR) were significant. Thus, for the entire group, SS vs. BR yielded $\mathrm{t}(23)=5.80, \mathrm{p}<.01$ and for SR vs. BR, $t(23)=4.86, p<.01$. For the highest eight subjects, the corresponding results were $t(7)=5.99$ and 7.49, respectively, with $p<.01$ in each case. Thus, the results of the first two experiments were replicated under binocular viewing, viz, luminance differences were present but without effect under conditions where blur significantly reduced the RFE.

\section{DISCUSSION}

Differences in peak luminance over a range of about $.68 \mathrm{log}$ unit were ineffective in altering the RFE. The insensitivity of the RFE to changes in luminance is consistent with the previously reported absence of effects of light intensity on the rod-and-frame test (Nyborg, 1972, 1974). Although the present study examined luminance levels about $2 \log$ units above scotopic threshold (about $5 \times$ $10^{-5} \mathrm{Nit}$, the results are consistent with the finding of
Leibowitz et al. (1979) of an absence of effect, even at scotopic luminance levels, on circularvection. In contrast with the innocuous role of blur in circularvection, however, the present results did show a highly significant dependence of the RFE on the magnitude of blur. This difference does not appear to be due to a difference in levels of blur used in the two studies since, in Leibowitz et al., the spatial frequency of the circularvection pattern was $.067 \mathrm{cpd}$ without the $16 \mathrm{D}$ refractive error. The latter would have reduced the spatial frequency somewhat further yet. In the present study, the lowest spatial frequency, which was .092 cpd in Condition HB, produced substantial reductions in RFE, although the effect remained robust, in that the RFE was not reduced to zero. This pattern of differences and common effects suggests that retinal blur, especially with respect to the high magnitudes employed, differentially affects sensitivity of static and dynamic orientation, the latter being less affected than the former. Apparently, the sensitivity of the orientation control system to low-spatial-frequency patterns in the retinal periphery is increased by motion.

The spatial-frequency dependency found in the present study suggests a neurophysiological basis for the control of static orientation-namely, that it is determined by the spatial characteristics of receptive fields in peripheral retina. These fields, in turn, serve as a source of stimulation to populations of highly selective orientation detectors, most likely influencing spatial orientation via pathways between the vestibular nucleus and oculomotor centers, and the vestibular cerebellum (Daunton \& Thomsen, 1979; Dichgans \& Brandt, 1972; Waespe \& Henn, 1977). These subcortical sites, at which visual-vestibular interactions take place, help to explain the noncognitive automatic, reflex-like character of the effect of the tilted frame on orientation perception.

Within the range of bandwidths employed in the present study, that is, from .092 to $.862 \mathrm{cpd}$, as represented in Figure 2, the RFE was facilitated at the higher spatial frequencies. High scorers, however, were more sensitive than low scorers to the changes in spatial frequency, as shown by the differences in the slopes of the fitted functions. This result offers the basis for an explanation of individual differences in RFE. If one assumes that these reflect corresponding differences in the extent of visualvestibular interactions, then a likely reason for this may be found in the size distribution of peripheral receptive fields. Presumably, the larger the receptive field, the more sensitive one is to low spatial frequencies, and the smaller the receptive field, the more sensitive one is to higher spatial frequencies. The peripheral retina of low scorers, therefore, may simply lack the wide receptive fields necessary to process the relatively low range of spatial frequencies examined in the present study.

Gender differences, commonly encountered in spatial orientation research, also may be reflected in the distribution of receptive field sizes in peripheral retina. Accordingly, one may expect individuals and genders for a given degree of retinal eccentricity to differ in the shape 
of the peripheral contrast sensitivity function, with males, for example, having a higher low-frequency cutoff than females. Alternatively, one may find, with increasing eccentricity, that males and low scorers tend to drop in sensitivity to high spatial frequencies at a lower rate than do females and high scorers.

\section{REFERENCES}

Daunton, N., \& Thomsen, D. (1979). Visual modulation of otolithdependent units in cat vestibular nuclei. Experimental Brain Research, 37, 173-176.

Dichgans, J., \& BRANDT, Th. (1972). Visual-vestibular interaction and motion perception. In J. Dichgans \& E. Bizzi (Eds.), Cerebral control of eye movements and motion perception (pp. 327-338). Basel: S. Karger.

DichGaNS, J., \& BRANDT, TH. (1978). Visual-vestibular interactions: Effects on self-motion perception and postural control. In R. Held, H. Leibowitz, \& H. L. Teuber (Eds.), Handbook of sensory physiology: (Vol. VIII) Perception. New York: Springer.

EBENHOLTZ, S. M. (1977). Determinants of the rod and frame effect: The role of retinal size. Perception \& Psychophysics, 22, 531-538.

Ebenholtz, S. M., \& Benzschawel, T. L. (1977). The rod and frame effect and induced head tilt as a function of observation distance. Perception \& Psychophysics, 22, 491-496.

Ebenholtz, S. M., \& Callan, J. W. (1980). Modulation of the rod and frame effect: Retinal angle vs apparent size. Psychological Research, 42, 327-334.

Ebenholtz, S. M., \& Glaser, G. W. (1982). Absence of depth processing in the large-frame rod-and-frame effect. Perception \& Psychophysics, 32, 134-140.

Leibowitz, H. W., Rodemer, C. S., \& Dichgans, J. (1979). The independence of dynamic spatial orientation from luminance and refractive error. Perception \& Psychophysics, 25, 75-79.

NyBORG, H. (1972). Light intensity and the perception of the vertical. Scandinavian Journal of Psychology, 13, 314-326.

NyBoRG, H. (1974). Light intensity in the rod-and-frame test reconsidered. Scandinavian Journal of Psychology, 15, 236-237.

Reason, J. T., Mayes, A. R., \& Dewhurst, D. (1982). Evidence for a boundary effect in roll vection. Perception \& Psychophysics, 31, 139-144.
Sigman, E., Goodenough, D. R., \& Flannagan, M. (1979). Instructions, illusory self-tilt, and the rod-and-frame test. Quarterly Journal of Experimental Psychology, 31, 155-165.

Streibel, M. J., Barnes, R. D., Julness, G. D., \& Ebenholtz, S. M. (1980). Determinants of the rod-and-frame effect: Role of organization and subjective contour. Perception \& Psychophysics, 27, 136-140.

WAESPE, W., \& HENN, V. (1977). Neuronal activity in the vestibular nuclei of the alert monkey during vestibular and optokinetic stimulation. Experimental Brain Research, 27, 523-538.

WITKIN, H. A., \& ASCH, S. E. (1948). Studies in space orientation: IV. Further experiments on perception of the upright with displaced visual fields. Journal of Experimental Psychology, 38, 762-782.

Witkin, H. A., \& Goodenough, D. R. (1981). Cognitive styles: Essence and origins. New York: International Universities Press.

\section{NOTES}

1. The Gaussian continues ad infinitum. The choice of $3 \sigma$ as the appropriate cutoff point was based on the calculation that at $3 \sigma$ luminance has fallen to $1.10 \%$ of peak amplitude. Given the values used in the present studies, this approximated levels at or below scotopic luminance thresholds.

2. In terms of an equivalent sinusoidal luminance modulation profile, low, medium, and high blur yielded spatial frequencies of .455 ,

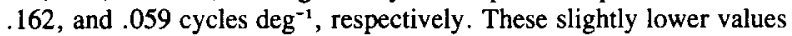
relative to the Gaussian modulation arise from the fact that, for a sine wave, the region spanned when the function falls to $1 / 2$ peak amplitude represents $50.0 \%$ of the total bandwidth. Hence, the bandwidth based on sinusoidal estimates is larger than that based on the Gaussian model.

3. This is taken as the inverse of the full bandwidth. Of course, the directly seen frame in Condition NB has harmonic components not present in the blurred patterns. Nevertheless, to maintain consistency in measurement, this condition is represented on the abscissa in Figure 2 in terms of the bandwidth derived from its photometrically determined luminance profile. If, however, only the three blur conditions were to be considered, the basic trend remains the same; viz, the RFE decreases with decreasing spatial frequency.

(Manuscript received March 23, 1984; revision accepted for publication December 10, 1984.) 